\title{
Admission Control Algorithm for MPLS-TE Networks
}

\author{
Ahmed S. Gab Allah \\ Faculty of Engineering \\ Tanta University \\ Tanta, Egypt
}

\author{
Nawal A. El-Fishawy \\ Faculty of Electronic \\ Engineering \\ Minoufiya University \\ Menouf, Egypt
}

\author{
Nada M. El-Shennawy \\ Faculty of Engineering \\ Tanta University \\ Tanta, Egypt
}

\begin{abstract}
Today real-time applications involve, Voice over IP (VoIP) is gaining increasing popularity. MPLS is developed to combine properties of Data-Link Layer with the flexibility and robustness of datagram Network Layer in efficient traffic transmission and QoS support. Connection Admission Control (CAC) mechanism for the RSVP-TE in MPLS network leaks its availability to achieve high performance especially with the increased importance of real-time application that need high end-to-end QoS. So, in this paper, Connection Admission Control (CAC) mechanism is proposed with inputs parameters not only bandwidth but also end-to-end delay and jitter to making its decision. A simulation comparison using OPNET simulator between the proposed algorithm and the most used CAC is presented. The results indicate that the proposed algorithm outperforms the other one.
\end{abstract}

\section{Keywords}

MPLS, Traffic Engineering, RSVP-TE, Admission Control.

\section{INTRODUCTION}

Today, real-time applications involve voice. Voice over IP (VoIP) and Video conferencing applications are gaining increasing popularity that is reinforced by the massive deployment of the wide range of fast access technologies. For supporting these applications efficiently, a new technique is required to serve these applications with less end-to-end delay and without need to increase bandwidth.

Multi-Protocol Label Switching (MPLS) technology is introduced to satisfy the real-time applications requirements in terms of Quality-of-Service (QoS) and Traffic Engineering[1], [2].Multiprotocol Label Switching (MPLS) can be used to improve the performance of the backbone networks[3]. The data packet can be switched on the basis of labels rather than routed on the basis of destination address by using MPLS; MPLS networks support different features such as Quality-of-Service (QoS) and Traffic Engineering (TE). TE is the operation which is used to select the best path for data traffic in order to balance the traffic load between the different links [4]. QoS is the ability to provide different priority to different applications, users, and data flows, to satisfy a certain level of performance to a certain application [5].

QoS is a very important issue when real time traffic flows are transported, because this type of application is more sensitive to delay and packet drops. The integration of QoS with the MPLS network enhances the performance of the network [6].

MPLS uses a signaling protocol named RSVP-TE to be able to use different labels for the same Label Switched path (LSP). RSVP is combined with the traffic engineering property in MPLS and it named RSVP-TE. It is an enhancement extension for the MPLS because it deals with end-to-end reservation of resources for traffic flows. RSVP-
TE is used as a label distribution protocol in traffic engineering MPLS networks. Network nodes that support both RSVP-TE and MPLS can attach labels with RSVP-TE flows [7],[8].

Connection Admission Control (CAC) mechanism is an essential part of RSVP-TE. CAC increases the availability of RSVP-TE to achieve high performance, especially for realtime applications, which need high end-to-end QoS. The authors in [9] introduced Fuzzy-CAC to work with dynamic traffic in the fast changing environment. Edge Admission Control is developed in [10] that computing admission thresholds and takes into account both the best-effort traffic's performance and QoS traffic's constraints. A routing-based admission control mechanism is presented for the IP traffic flows in MPLS networks, routing and admission control problem with multi-constrained end-to-end QoS is used in the presented CAC to improve MPLS networks [11].

\section{MULTI-PROTOCOL LABEL SWITCHING}

MPLS is an advancing technology that is combined the layer 3 as a packet switching technology with supporting Layer 2 protocols. MPLS addresses this feature by using a label for forwarding packets rather than IP address. The labels are added to the packet when it enters to the MPLS domain at ingress router then packets are forwarded through intermediate routers based on their labels by using a label-tolabel mapping technique. When the packets arrive to egress router, which is the outdoor router of the MPLS domain, the packet label is removed and its IP address will be used to send the packet to its destination node. MPLS has an architecture, which supports its functions. In this paper, the MPLS architecture is divides into three parts: MPLS Definition, MPLS header, and MPLS signaling protocols.

\subsection{MPLS definitions}

MPLS has some important definitions: Label Switch Router (LSR), Label Edge Router (LER), Forward Equivalence Class (FEC), and Label Switched path (LSP).

LSR: is a generic router which is placed in the MPLS domain and it is used to route the packets based on their labels. When LSR receives a packet, it will make a look up operation in its label's table using the packet label to find the next hop for that packet and its new label and change the old label by the new label then forward the packet.

LER: is a router which located in the edge of the MPLS domain. It is responsible for adding the first label to the incoming packet, here this router is called ingress router When the edge router function is removing the label from the outgoing packet from the MPLS domain, it is call egress router. 
FEC: FEC is a group of packets, which have the same properties, such as destination address, and priority.LSP: is the sequence of LSRs that is used to transfer packets, which have the same FEC.

Label Distribution Protocol (LDP): enables the exchange of labels between label switch routers (LSRs) in an MPLS network to binding label information database for supporting hop-by-hop forwarding.

\subsection{MPLS header}

As shown in Fig. 1, the MPLS label is added after the IP packet header; this label is called MPLS shim header. The MPLS header consists of 32-bit, and its fields are: Label, Experimental (EXP), S, and TTL (Time-to-Live).

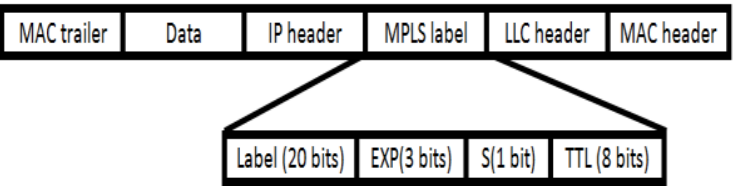

Fig. 1 MPLS Shim Header

Label is 20-bit address and it is used to forward the packet in the MPLS domain. EXP is a 3-bit which is used to indicate the packet QoS level. S is 1-bit, and it is used in nested MPLS domains. TTL consists of 8-bit to indicate the packet life time in the network.

\subsection{MPLS-TE signaling protocols}

MPLS-TE uses several types of control protocols like signaling protocols, which are used to distribute label information between nodes. The often used signaling protocols are Resource Reservation Protocol-Traffic Engineer (RSVP-TE) and Constraint-Based LSP Setup Using LDP (CR-LDP)

\subsubsection{RSVP-TE}

RSVP-TE [7] is the main signaling protocol, which is suitable for label distribution in MPLS-TE. It works with end-to-end reservation of resources for traffic flows. RSVP-TE has many facilities to describe traffics. It is upgraded to cover the main points in TE, such as label management, requesting and controlling routes, preempting resources, and maintaining connectivity between RSVP-TE LSRs.

\subsubsection{CR-LDP}

CR-LDP [12] uses LDP database for establishing end-to-end LSPs through MPLS-TE network under a set of some constraints. These constraints may be applied by applications, which require LSP or are required by users. CR-LDP has two main types of constraints: explicit routes and traffic parameters. CR-LDP has additional feature for supporting priority in assigned LSPs.

\section{MPLS CONNECTION ADMISSION CONTROL}

MPLS-TE supports CAC mechanism to manage and organize the applications with respect to their traffic requirements. CAC is an essential part for any network, which should attain QoS requirements. These requirements include: End-to-End delay, delay variation, jitter, and packet loss rate. The QoS requirements differ based on the application types, but these requirements are sensitive to real time applications: voice, video, etc. and have less importance for non-real time applications [13]. CAC is important for supporting QoS requirements in both access and backbone networks

In MPLS-TE, CAC functions are located at the edge router, and it works when anew LSP needs setup. CAC functions are responsible for determining if the new LSP can be accepted without violating existing QoS connections and the network throughout requirements. The most used CAC in MPLS is Generic Connection Admission Control (GCAC).

\subsection{Generic connection admission control}

The GCAC in RSVP-TE in MPLS is required to take a decision of setup LSP or rejected based on its inputs. GCAC is designed to take only bandwidth as input.

LER receives a connection request including the requested bandwidth .It follows specific procedure 1) determines whether there is enough bandwidth on all LSR through LSP from ingress to egress node, 2) ingress router reception of RESV message setup LSP if all LSR have enough bandwidth 3 ) keeps tracking of network resources. The technique behind the MPLS GCAC is that the ratio between the bandwidth margin that the network node is put above the sustained bandwidth and the standard deviation of the sustained bandwidth does not change significantly, as one new aggregate flow is added on the link [14]

\section{PROPOSED CAC ALGORITHM}

With increase of the used real-time application over networks, supporting QoS requirements will be important to increase the network performance. CAC is an important part for QoS. So, in this paper CAC is developed and passed on not only the bandwidth but also on delay and jitter of applications in order to enhance the performance of the real-time application and improve the network performance by ensure the resources distribution and minimum delay. The proposed CAC is divided into two tiers, Fig. 2.

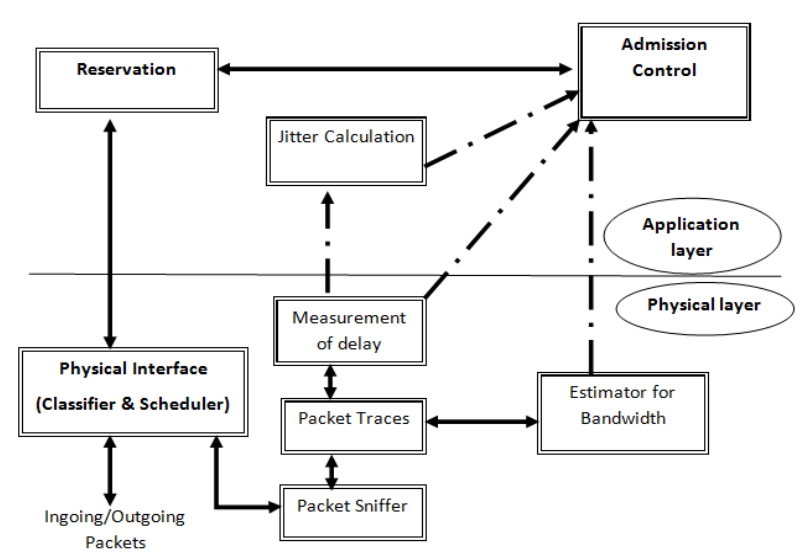

Fig. 2 Proposed CAC Structure

\subsection{Calculation tier}

The computation of the CAC used parameters is done. Packets delay and jitter are computed. These parameters are used with the estimated bandwidth as inputs to the next tier to help the CAC to take its decision about the new LSP establishment. 


\subsection{Take decision tier}

Based on the calculated delay, jitter and the requested bandwidth, the proposed CAC takes its decision for creating or rejecting the new LSP by applying some check rules.

First bandwidth check is to determine that the new LSP can work in the network without any effects on the bandwidth of the other running traffics:

Bandwidth ${ }_{\text {Request }} \leq$ Bandwidth $_{\text {available }}$

If the bandwidth check is TRUE, CAC goes to check the delay constraint:

Computed delay $\leq$ delay $_{\max }$

If the delay check is TRUE, CAC goes to check the jitter constraint:

Computed jitter $\leq$ jitter $_{\text {max }}$

Where the delay max and jitter max are the maximum accepted delay and jitter for the requested traffic type. These values are standard for all types of applications. Bandwidth ${ }_{\text {available }}$ is the available bandwidth of the network

If all conditions are True, new LSP will be established. In False case of any condition checking, the creation request of LSP is rejected.

\section{SIMULATION METHODOLOGY}

To evaluate the performance of the proposed algorithm the OPNET Simulator 14.5[15] is used. OPNET is a real-time network simulator which designed mainly for the evaluation and analysis of network models

The used network model [16] is shown in Fig. 3. The network contains of: six LSRs [LSR 1,LSR 2,LSR 3,LSR 4,LSR 5, and LSR_6], Two LERs [East_LER, and Weast_LER], these routers are connected by PPP_adv link work at data rate of $5 \mathrm{Mbps}$, seven clients [Voice_caller, Voice_called , Video_caller, Video_called, FTP_client, HTTP_client, and Email_client], two switches [East_SW, and Weast_SW] and three servers for the used applications [ FTP_server , HTTP_server, Email_server ].

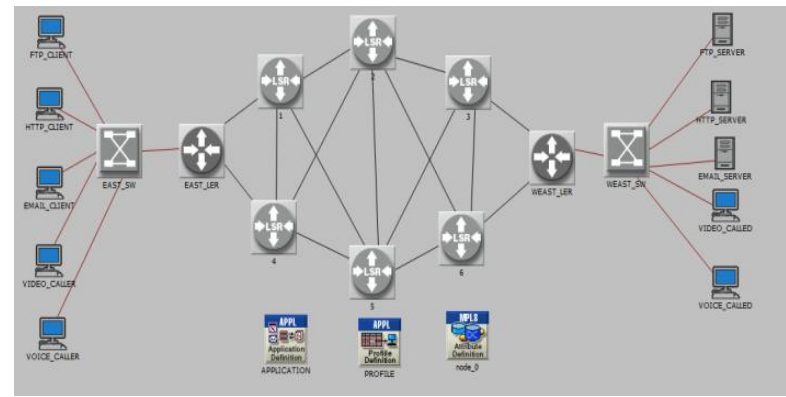

Fig. 3 The MPLS Network Topology

The simulation time for each scenario is 600 seconds. The best and the worst case scenarios were applied in the current research. The best case scenario defines the link under test to be completely empty at the starting point of the testing, increasing load by initiate application sequentially. The worst case scenario characterizes has $60 \%$ background traffic flow from beginning of simulation. In the performance study of the proposed algorithm compares with GCAC, and jitter, end-toend delay, delay variation, and traffic received for each application are used as performance metrics.

\section{RESULTS AND ANALYSIS}

The evaluation of the proposed CAC compared with GCAC is introduced based on jitter, end-to-end delay, delay variation, and traffic received for each application.

\subsection{Voice application}

The performance of voice application is presented based on the change of CAC type.

Fig. 4, shown the effect of the proposed algorithm in voice jitter, as indicated from the figure, the Proposed CAC and GCAC approximately have same jitter value until time equal to $450 \mathrm{~s}$, the jitter value of the proposed algorithm decreased to $-0.2 \mu \mathrm{s}$.

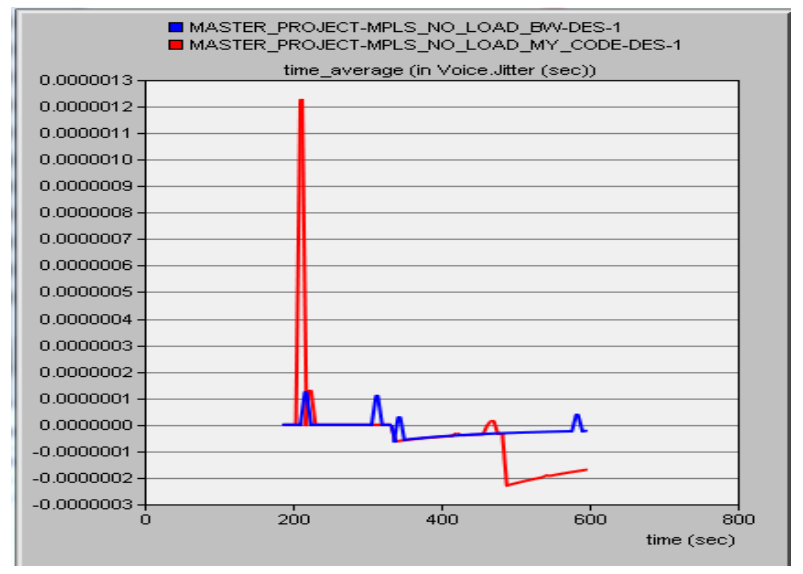

Fig. 4 The voice jitter-Best scenario

The voice packet delay variation and end-to-end delay are shown in Fig. 5 and Fig. 6, respectively.

From Fig. 5, we can see that the Proposed CAC has the highest voice packet delay variation of about $39,38 \mu \mathrm{s}$ at the end of the simulation, whereas GCAC has $35.41 \mu$ s with difference $3.97 \mu$ s .

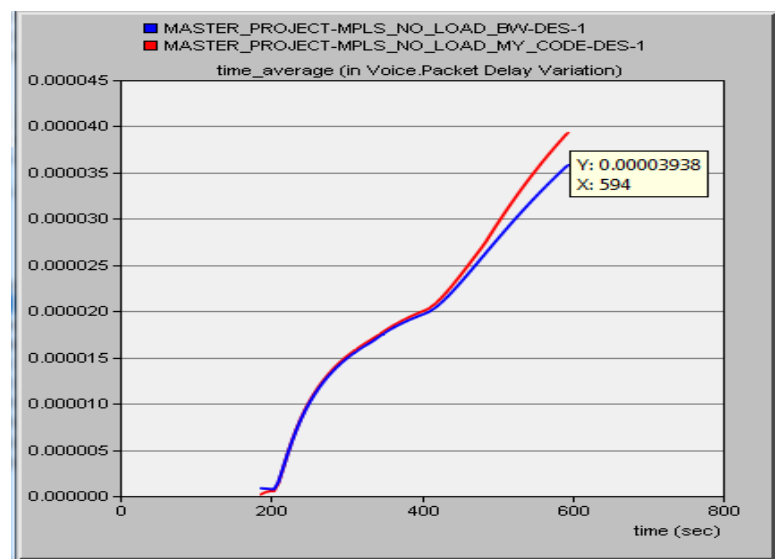

Fig. 5 The Voice delay variation-Best scenario

Fig. 6 shows that the end-to-end delay value of the proposed algorithm and GCAC are the same until nearly $450 \mathrm{~s}$, the Proposed CAC gently increase than GCAC by $0.35 \mathrm{~m} \mathrm{~s}$. 


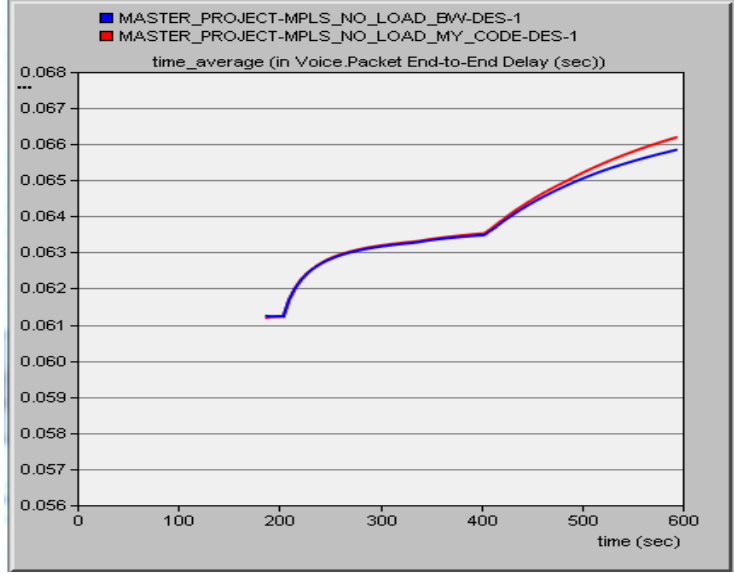

Fig. 6 The voice end-to-end delay-Best scenario

An important node can be seen from the results: the increasing of the delay variation, jitter and end-to-end delay don't exceed the maximum threshold value of delay and jitter. This increase is due to other traffic in the network. The reason for this situation can be due to the high traffic load of the other.

In the scenario of a worst case network, the proposed CAC improve its performance and it outperforms the other protocol in respect to jitter as shown in Fig. 7.

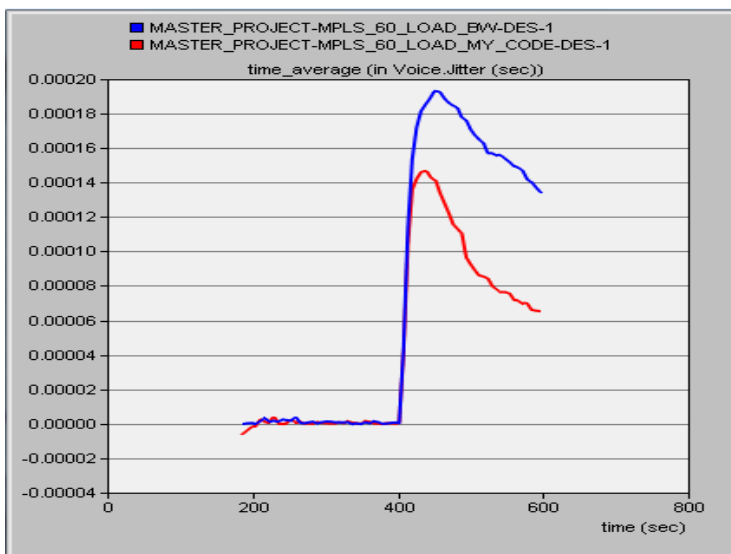

Fig. 7 The Voice jitter-Worst scenario

Packet delay variation is represented in Fig. 8, Proposed CAC is greater than GCAC, whereas after $500 \mathrm{~s}$ from beginning of simulation the delay of the proposed CAC is decreasing significantly.

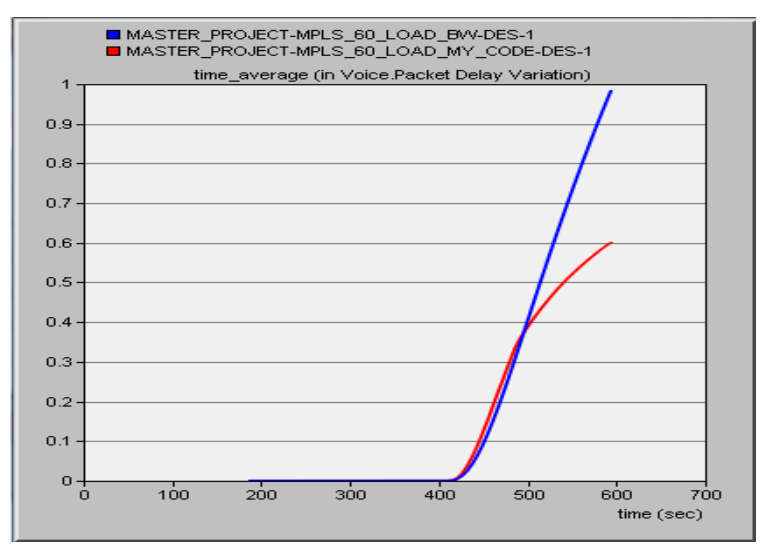

Fig. 8 The voice delay variation- Worst scenario
From the Fig. 9, it is noticed that end-to-end delay in proposed CAC has lower value, after $500 \mathrm{~s}$ from simulation time.

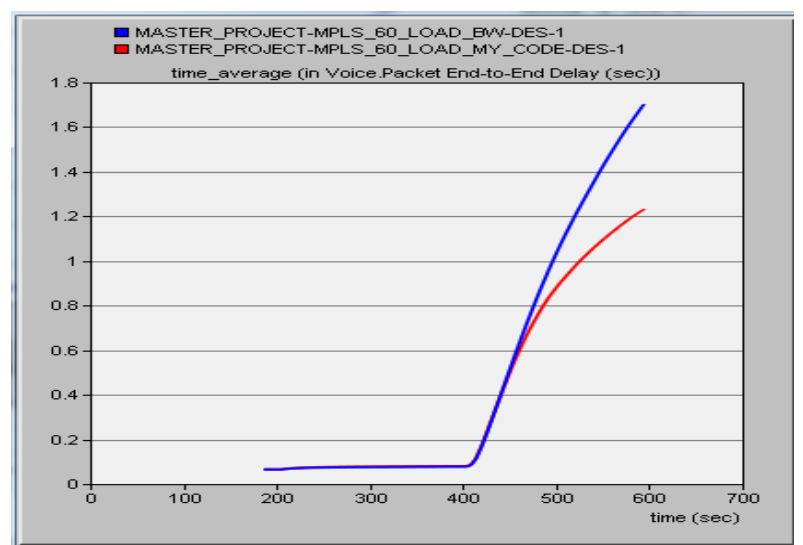

Fig. 9 The voice end-to-end delay- Worst scenario

From the voice results, we can notice that the Proposed CAC improve the utilization of voice application by increase the traffic received with slightly rise in jitter, delay variation, and end-to-end delay as shown in Table 1.

Table 1. Voice Result Details

\begin{tabular}{|c|c|c|c|c|}
\hline \multirow{2}{*}{} & \multicolumn{2}{|c|}{ Best Case } & \multicolumn{2}{c|}{ Worst Case } \\
\cline { 2 - 5 } & $\begin{array}{c}\text { Threshold } \\
\text { CAC }\end{array}$ & $\begin{array}{c}\text { Proposed } \\
\text { CAC }\end{array}$ & $\begin{array}{c}\text { Threshold } \\
\text { CAC }\end{array}$ & $\begin{array}{c}\text { Propos } \\
\text { ed } \\
\text { CAC }\end{array}$ \\
\hline Jitter & $-.023 \mu \mathrm{s}$ & $-.17 \mu \mathrm{sec}$ & $134.8 \mu \mathrm{sec}$ & $65.4 \mu \mathrm{s}$ \\
\hline $\begin{array}{c}\text { Delay } \\
\text { variation }\end{array}$ & $35.87 \mu \mathrm{s}$ & $\begin{array}{c}39.38 \\
\mu \mathrm{sec}\end{array}$ & $.98 \mathrm{sec}$ & $.6 \mathrm{~s}$ \\
\hline $\begin{array}{c}\text { End -to- } \\
\text { end delay }\end{array}$ & $65.85 \mathrm{~m} \mathrm{~s}$ & $66.2 \mathrm{~m} \mathrm{~s}$ & $1.7 \mathrm{~s}$ & $1.2 \mathrm{~s}$ \\
\hline $\begin{array}{c}\text { Packet } \\
\text { drop }\end{array}$ & $18.8 \%$ & $0 \%$ & $25 \%$ & $11 \%$ \\
\hline
\end{tabular}

\subsection{Video application}

Proposed CAC improve the utilization of video application by increase the traffic received ratio after $400 \mathrm{~s}$ (the second video call), whereas rising in delay variation, and end-to-end delay.

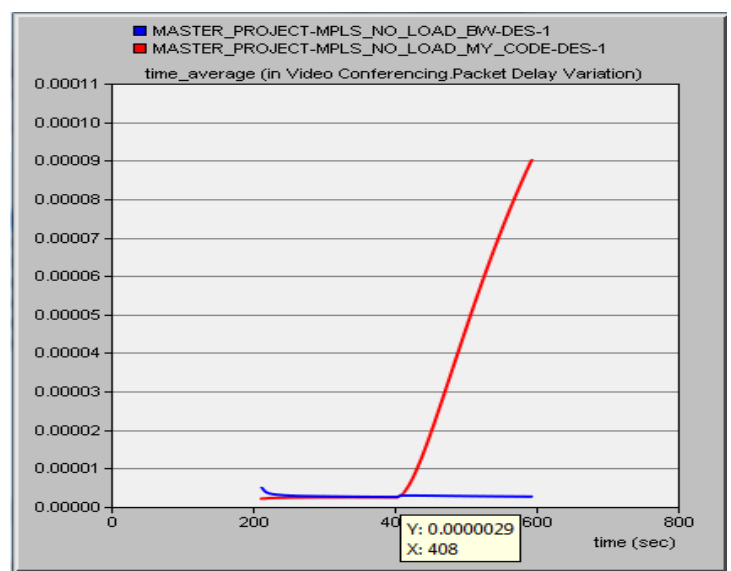

Fig. 10 The video delay variation-Best scenario 
Fig. 10 indicates that the delay variation for the GCAC started to increase from $2.15 \mu \mathrm{s}$ at $200 \mathrm{~s}$ after the start of the simulation to about $90.5 \mu \mathrm{s}$ at the end of the simulation. This increase was rapid after $400 \mathrm{~s}$ (second video call started) from start of simulation. Also, end-to-end delay remains in the no change state and equal until $400 \mathrm{~s}$, then increased due to traffic congested, Fig. 11.

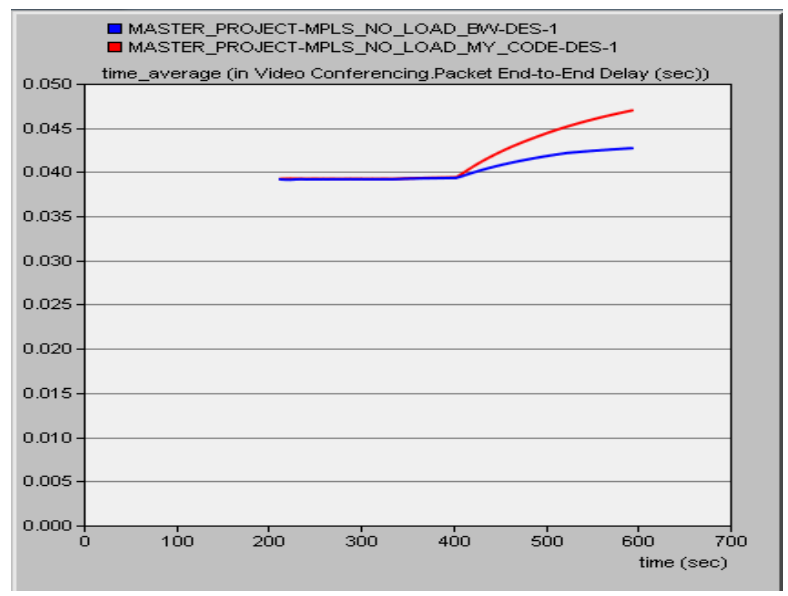

Fig. 11 The video end-to-end delay-Best scenario

In the scenario of worst case, the proposed CAC improves its performance, because the rejection of the second LSP of video call which cause more free bandwidth to use by the running applications. As seen in Fig. 12, delay variation is increasing slightly, but by the end of simulation the two protocols have the same value of delay variation.

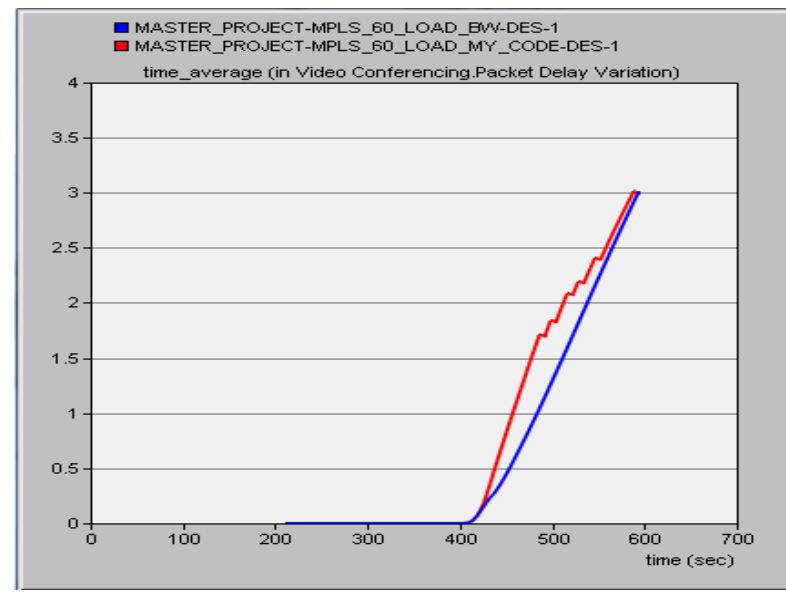

Fig. 12 The video delay variation- Worst scenario

For video end-to-end delay, it is decreasing after $450 \mathrm{~s}$. as shown in Fig. 13. TheProposed CAC saves the network from congest according to the results shown in table 2, and it rejects the new flow creation. Hence, the overall network performance improvement will be resulted.

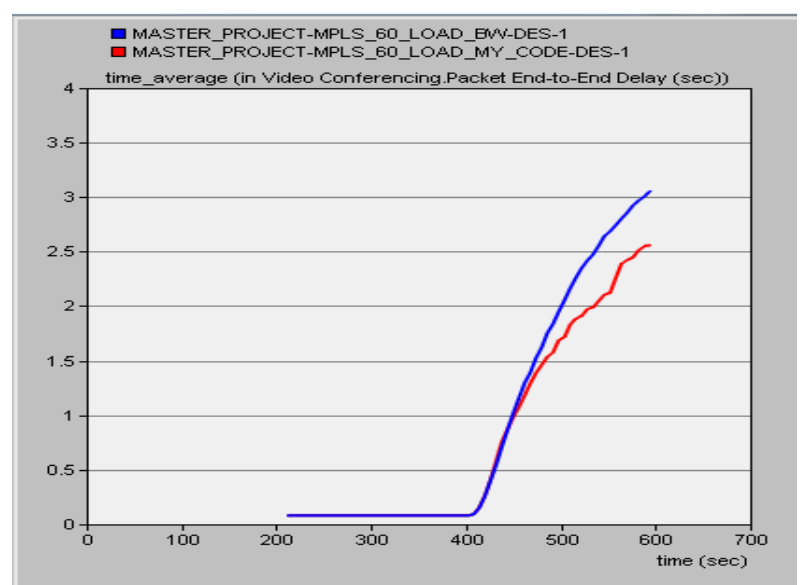

Fig. 13 The video end-to-end delay- Worst scenario

Table 2. Video Result Details

\begin{tabular}{|c|c|c|c|c|}
\hline \multirow{2}{*}{} & \multicolumn{2}{|c|}{ Best Case } & \multicolumn{2}{c|}{ Worst Case } \\
\cline { 2 - 5 } & $\begin{array}{c}\text { Threshol } \\
\text { d }\end{array}$ & $\begin{array}{c}\text { Proposed } \\
\text { CAC }\end{array}$ & Threshold & Proposed \\
CAC & CAC \\
\hline $\begin{array}{c}\text { Delay } \\
\text { variation }\end{array}$ & $2.7 \mu \mathrm{s}$ & $90 \mu \mathrm{s}$ & $3.02 \mathrm{~s}$ & $3 \mathrm{~s}$ \\
\hline $\begin{array}{c}\text { End -to- } \\
\text { end delay }\end{array}$ & $42.7 \mathrm{~m} \mathrm{~s}$ & $47 \mathrm{~m} \mathrm{~s}$ & $3.06 \mathrm{~s}$ & $2.55 \mathrm{~s}$ \\
\hline $\begin{array}{c}\text { Packet } \\
\text { drop }\end{array}$ & $4.7 \%$ & $0 \%$ & $53.6 \%$ & $57.6 \%$ \\
\hline
\end{tabular}

\section{CONCLUSIONS}

Nowadays, real-time applications such as: voice especially voice over IP (VoIP) and Video conferencing applications are gaining an increasing popularity, reinforced by the massive deployment of the wide range of fast access technologies. MPLS-TE technology is developed to satisfy these applications requirements in terms of Quality-of-Service (QoS) and traffic engineering (TE).MPLS-TE supports CAC mechanism to manage and organize the applications with respect to their traffic requirements. CAC is an essential part for any network which should meet QoS requirements. In this paper, A CAC algorithm is proposed and it is making its decision based on bandwidth, delay, and jitter. So, it provides service quality for real-time applications. The simulation results of the proposed algorithm that are compared with other algorithm are shown that the proposed algorithm outperforms the other one with respect to delay, jitter, and delay variation. The future research expects a dynamic CAC can improve the threshold value to be dynamically modifying its parameters based on network condition to achieve optimal decision making under different network conditions. 


\section{REFERENCES}

[1] Aggarwal, J. and Dhall, A. "Performance Analysis of MPLS over VOIP" International Journal of Science, Engineering and Technology Research (IJSETR), 2015, Volume 4, Issue 6, pp.1896-1901.

[2] Kharel, J., and Adhikari, D. 2011. Performance Evaluation of Voice Traffic over MPLS Network with TE and QoS Implementation. M.S. thesis,Dept. Electrical Engineering, School of Computing at Blekinge Institute of Technology, Sweden .

[3] Kale , N. N. ,and Waichol, S. A. "Performance Analysis of MPLS network with Traditional IP Network in Service Provider Environment" International Journal of Advanced Research in Computer Engineering \& Technology (IJARCET),Volume 3, Issue 4, pp.13111316, 2014

[4] Kumar, M. j. , and Sangal, S. "Improving the usage of Network Resources using MPLS Traffic Engineering (TE) " International Journal of Current Engineering and Technology, Vol.5, No.1, pp.261-265, 2015.

[5] Evans, J., and Filsfils, C. 2007, Deploying IP and MPLS QOS for Multiservice Networks Theory and Practice, 1st ed. , San Francisco , El-SEVIER.

[6] Abouseda, M. I., Bozed, A., and Zerek, A. R. 2015 .Comparative study of QoS and performance of VoIP using MPLS-TE network. 16th International Conference on Sciences and Techniques of Automatic Control and Computer Engineering (STA), pp. 784-788.

[7] Awduche, D., and et al. "RSVP-TE: Extensions to RSVP for LSP Tunnels" Network Working Group RFC 3209( 2001)

[8] Akinsipe, O. G., and Akinsipe, O. O. "Evaluating IP, MPLS and MPLS RSVP-TE Networks" Communications on Applied Electronics (CAE) - ISSN:
2394-4714 Foundation of Computer Science FCS, New York,USA ,Volume 3 - No.1, pp.28-31, 2015.

[9] Jelinskis, J., and Skrastins, A. 2014 .Analysis of Effective Fuzzy-CAC Solution for Proactive Traffic Engineering. 22nd Telecommunications forum TELFOR, pp.119-122.

[10] Meng, Z., Zhang, L., and Zhou, J. "EDGE ADMISSION CONTROL IN MPLS NETWORK" IEEE, pp.213-216, 2009.

[11] Oulai, D., Chamberland, S., and Pierre, S. "A New Routing-Based Admission Control for MPLS Networks" IEEE COMMUNICATIONS LETTERS, VOL. 11, NO. 2, pp.216-218, 2007.

[12] Jamoussi, B. and et al. "Constraint-Based LSP Setup using LDP" Internet Engineering Task Force (IETF), RFC 3212, 2002.

[13] Sulaiman, A. R., and SalihAlhafidh, O. Kh "Performance Analysis of Multimedia Traffic over MPLS Communication Networks with Traffic Engineering" International Journal of Computer Networks and Communications Security, VOL.2, NO. 3 , pp.93-101, 2014.

[14] Ash, G., Ed., and McDysan, D. "Generic Connection Admission Control (GCAC) Algorithm Specification for IP/MPLS Networks" Internet Engineering Task Force (IETF), RFC 6601, 2012.

[15] Opnet Product Documentations, Version 14.5, OPNET Inc.

[16] Arora, N. ,and Kaur, S. "Performance Evaluation and Fault Repair Mechanism for Resilience of MPLS RSVPTE Network with different application Traffic load balancing across Label Switched Path" International Journal of Engineering Technology Science and Research, Volume 2, Issue 11, pp.28-33 , 2015. 